\title{
Direct Comparison of Tungsten Nanoparticles and Foils under Helium Irradiation at High Temperatures Studied via In-Situ Transmission Electron Microscopy
}

\author{
E. Aradi ${ }^{1}$, J. Lewis-Fell ${ }^{1}$, R.W. Harrison ${ }^{2}$, G. Greaves ${ }^{1}$, A.H. Mir ${ }^{1}$, S.E. Donnelly ${ }^{1}$ and J.A. Hinks ${ }^{1 *}$ \\ 1. University of Huddersfield, Queensgate, Huddersfield, HD1 3DH, United Kingdom \\ 2. University of Manchester, Sackville Street, Manchester, M1 3NJ, United Kingdom \\ * Corresponding author: j.a.hinks@hud.ac.uk
}

The nanoengineering of materials for enhanced radiation damage tolerance by increasing the density of defect sinks and recombination centres has been investigated in nanograined [1], nanolayered [2], nanoporous [3] and nanodispersion-strengthened [4] materials. For example, in a nanoporous material an interconnected network of ligaments forms a structure in which the surface-area-to-volume ratio, $R_{\mathrm{SV}}$, is high and the distance to the nearest surface is always short. These surfaces act as insaturable sinks at which defects can annihilate and mobile gas atoms escape. This is particularly important in nuclear materials where neutron irradiation can induce the creation of vacancies and interstitials via atomic displacements as well as the introduction of insoluble gases such as helium from $(n, \alpha)$ reactions.

The low sputter yield, high thermal conductivity $\left(174 \mathrm{~W} \cdot \mathrm{m}^{-1} \cdot \mathrm{K}^{-1}\right.$ at $\left.300 \mathrm{~K}\right)$ and melting temperature $\left(3422^{\circ} \mathrm{C}\right)$ of tungsten have resulted in it being considered as the lead candidate for the plasma-facing material in the divertor of the ITER magnetic-confinement nuclear fusion reactor under construction in France [5]. The role of the divertor is to extract the ash (waste helium) produced by deuterium-tritium fusion reactions in the plasma. In doing so, it will be exposed to helium ions and $14.1 \mathrm{MeV}$ neutrons escaping from the plasma resulting in the accumulation of atomic displacements and helium leading to embrittlement, swelling and thermal conductivity changes [6].

Each ligament in a nanoporous structure can be considered as an individual nanoparticle. To enable exposure to identical experimental conditions and thus direct comparison, electron-transparent foils of tungsten were decorated with tungsten nanoparticles to create specimens containing these two systems with significantly different values of $R_{\mathrm{SV}}$ (for example, for a $50 \mathrm{~nm}$ thick foil and $50 \mathrm{~nm}$ nanoparticle $R_{\mathrm{SV}}$ would be 0.04 and 0.12 , respectively). These were heated to either $500^{\circ} \mathrm{C}$ or $750^{\circ} \mathrm{C}$ and irradiated with $15 \mathrm{keV}$ helium ions and studied in-situ via transmission electron microscopy (TEM) in the Microscope and Ion Accelerator for Materials Investigations (MIAMI-2) system at the University of Huddersfield outlined in figure 1(a). The numbers of displaced atoms and implanted helium ions have been calculated using the Transport and Range of Ions in Matter (TRIM) Monte Carlo computer code [7]; whilst this can easily be done for the foil regions of the specimen it is not directly applicable to spherical nanoparticles as, natively, TRIM considers only planar geometries. Therefore, an in-house implementation of TRIM called Spherical Ion Calculation Modifier (SICMod) has been developed which takes the three-dimensional atomic collision cascades calculated by TRIM, distributes their entry points over the irradiated surface of the nanoparticle and then determines how much of each cascade would have been contained within the sphere and which branches would have terminated at the surface.

As shown in the example in figure $1(\mathrm{~b})$, at $500^{\circ} \mathrm{C}$ black spot radiation damage was observed to readily form in the foils but this was not the case in the nanoparticles to the end fluence of $5.0 \times 10^{17}$ ions. $^{-2}$. At $750^{\circ} \mathrm{C}$ in figures $1(\mathrm{c}, \mathrm{d})$, bubbles were found in both the foils and the nanoparticles but in the latter they were larger with a lower areal density compared to areas of the foil with thickness corresponding to 
an equivalent volume of material. These results point to the enhanced annihilation of defects at the surface of the nanoparticles which prevents the clustering of point defects and thus reduces the number of nucleation sites (i.e. vacancies and vacancy complexes) for bubbles leading to fewer bubbles which each absorb a greater share of the available helium and vacancies compared to those in the foil $[8,9]$.

\section{References:}

[1] O. El-Atwani et al. J. Nucl. Mater. 458 (2015) p216

[2] E.G. Fu, A. Misra, H. Wang, L. Shao and X. Zhang J. Nucl. Mater. 407(3) (2010) p178

[3] E.M. Bringa et al. Nano Lett. 12(7) (2012) p3351

[4] J. Brodrick, D.J. Hepburn and G.J. Ackland J. Nucl. Mater. 445(1-3) (2014) p291

[5] T. Hirai et al. J. Nucl. Mater. 9 (2016) p616

[6] R.W. Harrison Vacuum 160 (2019) p355

[7] J.F. Ziegler, M.D. Ziegler and J.P. Biersack Nucl. Instrum. Methods B 268(11-12) (2010) p1818

[8] E. Aradi et al. Nanomater. 8(12) (2018) p1052

[9] Funded by the EPSRC under grants EP/M028283/1 and EP/M01858X/1
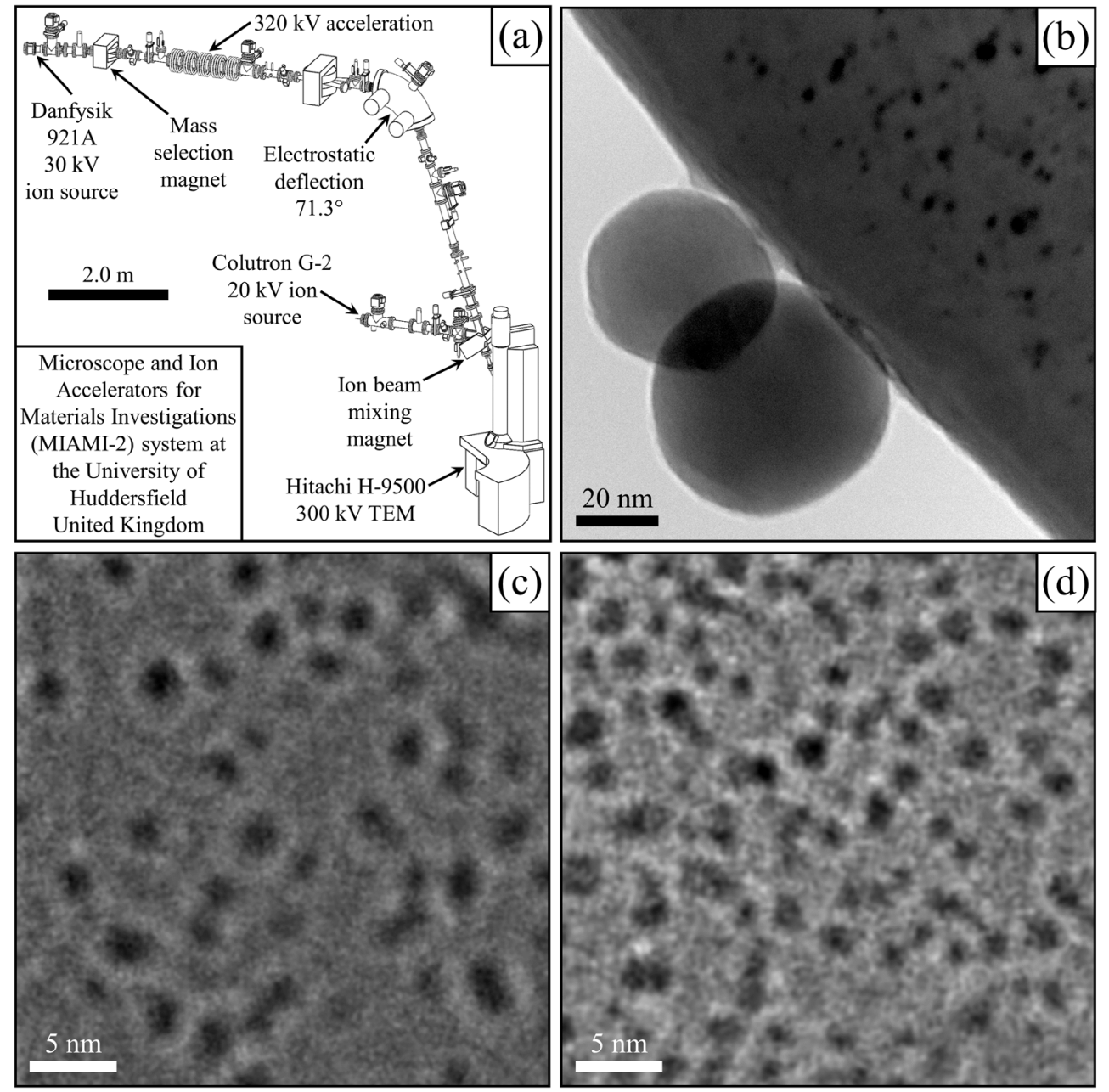

Figure 1. (a) Outline of the MIAMI-2 system incorporating dual ion-beamlines and a TEM; (b) underfocus TEM image of two nanoparticles (45 and $57 \mathrm{~nm}$ ) on a foil irradiated to $5.0 \times 10^{17}$ ions. $\mathrm{cm}^{-2}$ at $500^{\circ} \mathrm{C}$; and $(\mathrm{c}, \mathrm{d})$ representative overfocus TEM images of bubbles in a $50 \mathrm{~nm}$ diameter nanoparticle and a $50 \mathrm{~nm}$ thick foil, respectively, irradiated to $1.0 \times 10^{17}$ ions. $\mathrm{cm}^{-2}$ at $750^{\circ} \mathrm{C}$. 